\title{
Antiemetic research: future directions
}

\author{
Ian Olver • Alexander Molassiotis • Matti Aapro • \\ Jørn Herrstedt • Steven Grunberg • Garry Morrow
}

Received: 21 October 2010 / Accepted: 22 October 2010 /Published online: 10 November 2010

(C) Springer-Verlag 2010

\begin{abstract}
Purpose and methods As a part of reviewing the Multinational Association of Supportive Care in Cancer (MASCC) antiemetic guidelines in Perugia in 2009, an expert group identified directions for future antiemetic research.

Results and conclusions In future trials, the prediction of nausea and vomiting may combine algorithms based on observed prognostic factors relating to the patient and the
\end{abstract}

I. Olver

Cancer Council Australia,

Sydney 2000, Australia

\section{A. Molassiotis}

The School of Nursing, Midwifery and Social Work,

The University of Manchester,

Manchester M13 9PL, UK

M. Aapro

Clinique de Genolier, Multidisciplinary Oncology Institute, Genolier, Vaud 1272, Switzerland

J. Herrstedt

Department of Oncology, Odense University Hospital, 5000, Odense, Denmark

S. Grunberg

Division of Hematology/Oncology, University of Vermont, Burlington, VT 05405, USA

G. Morrow

University of Rochester School of Medicine and Dentistry

Rochester,

New York, NY 14642, USA

I. Olver $(\bowtie)$

Cancer Council Australia,

GPO Box 4708, Sydney, New South Wales 2001, Australia

e-mail: ian.olver@cancer.org.au anticancer therapy, the identification of the genes that code for receptors, and pharmacogenetic studies of the metabolism of drugs. Design issues for future trials include standardising the emetic stimulus across studies and finding the minimum tolerated effective dose and schedule of an antiemetic. Also control of delayed emesis is not independent of the control of acute emesis. The full range of side effects and the impact on global quality of life scores should be part of the routine assessment of an antiemetic. With current high rates of control of acute vomiting, future trials will need to consider new primary endpoints such as nausea, a complex symptom, where improvement is needed. Economic endpoints should be incorporated to ascertain the cost benefit of antiemetic prophylaxis, taking into account the impact of nausea on work capacity. New antiemetic drugs may be targeted at different receptors, such as opioid, cannabinoid and peptide YY receptors. New research is needed into determining the extent of corticosteroid use. The emetic potential of a range of newer cytotoxics particularly when used in combinations and different scheduling, such as prolonged oral dosing of cytotoxics and use of targeted therapies, are all areas in need of research. More antiemetic studies are needed in niche areas such as in patients receiving high dose chemotherapy, radiation therapy or combined modality therapy. Further evidence of the efficacy of newer antiemetic agents is required in children.

Keywords Antiemetics · Future research · Methodology · Chemotherapy $\cdot$ Nausea $\cdot$ Vomiting

\section{Introduction}

In 2009, a meeting was held in Perugia in Italy to revise the MASCC antiemetic guidelines. Experts review the litera- 
ture and produce guidelines according to the available evidence or by consensus if the evidence is poor. One committee is charged with identifying areas where future research would be most fruitful.

Although the control of both acute and delayed vomiting after anti-cancer chemotherapy is satisfactory in the majority of patients due to the combination of a 5-hydroxytryptamine 3 receptor $\left(5-\mathrm{HT}_{3}\right)$ antagonist, dexamethasone and a neurokinin 1 receptor $\left(\mathrm{NK}_{1}\right)$ antagonist, there are still specific areas requiring further research. With gene arrays and pharmacogenomics, we should have an opportunity to create better algorithms for predicting emesis. Nausea, a term which covers subjective feelings, is not controlled as well as vomiting. Data on emesis risk and the need for antiemetic prophylaxis have not been well researched with new drugs, targeted therapies and prolonged oral therapy. New receptors are targets for new antiemetic drugs and functional imaging will refine dosing strategies. We need to improve our creation and dissemination of guidelines.

\section{Prognostic factors}

Factors predicting who would vomit after chemotherapy have been identified over decades of antiemetic studies. The emetic potential of the drugs in the chemotherapy regimen, which in turn depends on their doses and schedules resulted in them being classified into groups of those with minimal, low, moderate or high risk of causing emesis if left untreated [18]. The emetic potential of combinations of drugs were more problematic to classify, due to the limited data available. For example, the combination of an anthracycline and cyclophosphamide, both of which have moderate emetic potential, results in a regimen of high emetic potential. Furthermore, the emetic risk classification does not include nausea.

There were also patient characteristics which were associated with a propensity to experience emesis post chemotherapy. There was more difficulty controlling postchemotherapy emesis in females, younger patients did not respond as well to antiemetics, and a history of prior alcohol intake of greater than $100 \mathrm{~g} /$ day was associated with less emesis following cisplatin chemotherapy [10, 38, $42,43]$. The reason for these observations could still be a useful area for further research. It is noteworthy, that addition of the $\mathrm{NK}_{1}$ receptor antagonist, aprepitant, to a combination of ondansetron and dexamethasone, seemed to negate the adverse prognostic effect of female gender on the prevention of chemotherapy-induced vomiting. With addition of aprepitant $66 \%$ of women and $69 \%$ of men were protected from emesis and did not need rescue medication after cisplatin-based chemotherapy, whereas only $41 \%$ of women as compared to $53 \%$ of men were protected without aprepitant [17].The physiological or pharmacological mechanisms of such gender differences, for example the influence of hormonal status, would be worth exploration.

Other factors being explored, are the susceptibility to motion sickness or the setting of the chemotherapy [32]. Furthermore, low emotional or role functioning, past history of morning sickness, anxiety, expectations for developing nausea/vomiting during chemotherapy or vestibular dysfunction have also been identified as key risk factors $[31,37,50]$. Further psychosocial factors need to be explored, as does the issue of whether the expectation of nausea and vomiting is correlated with its occurrence [19, 35].

Although clinical decision-making may be aided by creating an algorithm based on these prognostic factors for emesis, new genetic factors being investigated may make the prediction of emesis and selection of antiemetics and their doses more accurate. The emergence of gene array technology identifying the genes coding for the $5-\mathrm{HT}_{3}$ and $\mathrm{NK}_{1}$ receptors may allow clinical correlations and more rational selection of antiemetic regimens for patients. For example, patients with genetic variations in the $5-\mathrm{HT}_{3 \mathrm{~B}}$ receptor gene might respond differently to antiemetic treatment with the $5 \mathrm{HT}_{3}$ receptor antagonists [46].

Pharmacogenetic studies investigating functional genetic polymorphisms involving serotonergic, dopaminergic and neurokinin systems may allow more rational drug and dose selection for individual patients. For example, the efficacy of antiemetic treatment with some $5 \mathrm{HT}_{3}$ receptor antagonists (e.g. tropisetron) depends on cytochrome P-450 2D6 (CYP2D6) genotype, and rapid and ultrarapid metabolisers could be undertreated [23]. Ultrarapid metabolisers could, however, benefit from treatment with a $5-\mathrm{HT}_{3}$ antagonist not dependent of the CYP2D6 enzymes (e.g. granisetron).

\section{Design issues in clinical trials}

The randomised controlled double blind parallel design has been the gold standard for antiemetic studies, and will continue to be so, but there are issues to be resolved in the design of future trials. For example, the studies will have to be controlled for the new prognostic factors suggested above.

Another issue is standardising the emetic stimulus across studies. Cisplatin at greater than $50 \mathrm{mg} / \mathrm{m}^{2}$ has been the standard upon which regimens of high emetic potential have been based, but there has been confusion about the combination of an anthracycline and cyclophosphamide, two drugs of moderate emetic potential, which in combination become a regimen of high emetic potential.

When studying delayed emesis the response rate to an antiemetic may be influenced by the control of the acute phase of emesis. In the pivotal studies of aprepitant as part of a triple antiemetic regimen with a $5 \mathrm{HT}_{3}$ receptor 
antagonist and dexamethasone, the $\mathrm{NK}_{1}$ receptor antagonist was always used in the acute phase and then continued to cover the delayed phase. Palonosetron with its longer duration of action also was commenced in the acute phase. The response in the acute phase does influence the control of delayed emesis [2].

Side effects that are particularly due to a new antiemetic can be difficult to ascertain when antiemetics are given in combinations and the side effects of the cytotoxic therapy are also present. It is important to broadly record side effects so that unexpected effects are not missed. It is only in recent trials, for example, that a link between the use of aprepitant and increases in neutropenia and febrile neutropenia have been reported although this has not been confirmed in other studies [13]. The measurement of quality of life gives an overall assessment of the impact on the antiemetic on the wellbeing of the patient. This is particularly important not only because it serves to balance the efficacy and toxicity of the antiemetic but also because emesis impacts on other aspects of quality of life such as fatigue, appetite and sleep disturbance [26].

With response rates in acute vomiting approaching $90 \%$ in chemotherapy-naïve patients with triple antiemetic prophylaxis, it will be increasingly difficult to demonstrate a clinically meaningful benefit for a new agent looking at the first course of chemotherapy only. Therefore it can be useful to monitor the entire course of chemotherapy (4-6 cycles), when a new drug is investigated. The cut off for clinically meaningful improvement in patient reported outcomes has been suggested as $10 \%$, with the lowest estimate being $7 \%[7,36]$. Different endpoints in future studies are desirable. The most obvious of these is nausea which is not as well controlled as vomiting [30]. Nausea is an entity which encompasses different subjective and objective experiences and needs to be better defined in order to address it properly in future studies [40].

In time it may be possible to incorporate functional endpoints such as the effective blockade of receptors as demonstrated by PET scan which may allow early prediction of efficacy, as discussed below.

Economic endpoints should be part of randomised trials of new antiemetics and combinations. These are best measured prospectively and design issues discussed with health economists. It is important to ascertain the minimal effective dosing and scheduling of antiemetics [5, 25, 52]. This not only improves the therapeutic index but the cost efficacy ratio.

Other design issues include the measurement of efficacy over multiple cycles to see if there is any change in efficacy of the antiemetic regimen over time. The methodological challenges of evaluating antiemetic therapy over multiple cycles are well documented [14]. These studies are best done in patients with tumour types which will minimise the dropout rate. Patients dropping out can change the balance of prognostic factors over time. Also, statistical techniques need to account for the antiemetic efficacy in a particular cycle being influenced by the response in the previous cycle [42]. There is also a need to specifically explore regimens which will rescue that small percentage of patients who do not respond to the established antiemetics. Such patients can be pre-randomised to a particular rescue regimen if required or entered onto a study if they fail to respond to an antiemetic regimen given as prophylaxis prior to the first cycle of therapy.

\section{Usage of corticosteroids}

Corticosteroids such as dexamethasone are mentioned by guidelines as prophylaxis against chemotherapy of low emetic potential when administered as monotherapy, and are used to prevent vomiting and nausea in either chemotherapy of moderate or high emetic potential due to their synergistic efficacy in combination with other antiemetics. In guidelines, the scheduling and dosing of corticosteroids is tailored by taking into account the therapeutic setting and not the patient's characteristics, which are of importance. However, the clinician must consider that their administration may be associated with a range of side-effects, which may increase when administered as part of multiple-day antiemetic regimens [48]. A recent survey investigating moderate to severe side-effects associated with dexamethasone administered for prophylaxis against delayed CINV after MEC included insomnia (45\%), gastrointestinal symptoms $(27 \%)$, agitation $(25 \%)$, increased appetite $(18 \%)$, weight gain $(17 \%)$, skin rash (15\%) and depression (7\%) [48]. For these reasons, there is a particular interest in reducing their administration in certain clinical situations and/or in certain subsets of patients. Thus recently one double-blind study and one open-label study in patients receiving palonosetron plus dexamethasone on day 1 randomly assigned to receive either placebo or dexamethasone on days 2 and 3 have shown that there is an opportunity to reduce the total corticosteroid dose with no loss of efficacy in delayed MEC, although some patients experience an increase of nausea on some periods of observation $[1,6]$.

It should be noted that present guidelines, based on the fact that steroids were not used on days 2 and 3 of aprepitant containing antiemetic regimes when patients received AC like chemotherapy do not advocate the use of steroids for prevention of delayed nausea, while the studies showed that up to $50 \%$ of the patients suffered from nausea. This paradox will need further study in the setting of single day antiemetic regimens, which are highly effective in emesis control, but also seem to lose on control of nausea over several days [16]. 


\section{New antiemetics}

There are some drugs such as olanzapine which act on multiple receptors and are only just being investigated for post-chemotherapy emesis. Olanzapine is a thienobenzodiazepine which acts on multiple receptors including dopamine, serotonin, adrenergic, muscurinic and histamine receptors and has shown antiemetic activity in small studies with serotonin antagonists and steroids in both acute, and delayed emesis [33, 44].

The advent of the $\mathrm{NK}_{1}$ receptor antagonists demonstrated the utility of functional imaging of $\mathrm{NK}_{1}$ receptors by PET scan [15]. Techniques are evolving which can not only visualise where the receptors are, but quantitate them, and this will enable non-invasive methods of studying the binding of $\mathrm{NK}_{\mathrm{I}}$ receptor antagonists [34]. Such functional imaging can be used in developing new agents such as $\mathrm{NK}_{1}$ receptor antagonists which bind with higher affinity and will have a longer duration of action [21]. PET scans with appropriate labelled markers can be used to investigate different neurotransmitters and address issues like the optimal duration of receptor occupancy for antiemetic efficacy or the relative importance in acute or delayed emesis of central as compared to peripheral receptors.

There are still many possibilities for new anti-emetic agents to be developed as new receptor families are further investigated. The mu opioid receptors, for example, may mediate an antiemetic response in humans [41]. The cannabinoid CB1-receptor agonists possess broad-spectrum antiemetic activity in animal models in both chemotherapy and radiation therapy induced emesis [8, 47]. The synthetic cannabinoids nabilone and levonantradol have proven antiemetic activity in humans but side effects limit their usefulness [45, 49]. Studies of peptide YY and its analogues and its binding sites may result in a new class of antiemetic strategies [24].

\section{New research}

\section{Nausea}

We have suggested nausea as one of the primary endpoints for future studies because it has a much higher impact on daily living than vomiting and because it will be possible to more easily measure improvements but is not well controlled by present antiemetic regimens. Nausea is often present without the presence of vomiting, and hence these two symptoms are related but distinct phenomena. However, the subjective side effect of nausea in its own right needs further study. As a side effect it has the three components of severity, duration and frequency, which should be reported. However nausea is a subjective sensation which can only be reported by the patients who have been found to be reporting a complex array of symptoms ranging from anorexia to indigestion with nausea perhaps being only part of a symptom cluster [30]. More qualitative research is required to define the range of symptoms that patients report as nausea, so that a range of treatments in addition to antiemetics can be employed if necessary. A recent study in rats found that hypothalamic ghrelin decreased markedly $24-48 \mathrm{~h}$ after cisplatin and that intracerebroventricular injection of ghrelin was able to reverse the cisplatin-induced decrease in food intake. Furthermore the decrease in ghrelin secretion seemed to be caused by stimulation of $5-\mathrm{HT}_{2}$ receptors, because a $5-\mathrm{HT}_{2}$ receptor antagonist was able to enhance ghrelin secretion and to suppress cisplatin-induced anorexia [51]. Nausea may have different targets that may require the identification of new and unique receptor pathways. This may provide insights into other symptoms such as anorexia which may cluster with it.

Time course and severity of emesis, secondary antiemetic and new drug delivery systems

It should be routine in the development of new anticancer agents that the intensity and duration of emesis is determined to optimise the use of prophylactic antiemetics. More recent drugs in common usage such as oxaliplatin and pemetrexed require better characterisation of their emetic potential in combination therapies, as indeed other commonly used combination chemotherapy agents. Hence there is a need for more observational prospective data characterising the course of nausea/vomiting over several cycles to obtain more information on the emetic potential of combination drugs, which will help in an improved classification of the emetic potential of these drugs. This is also true for low and minimally emetic chemotherapy, where the data available is insufficient to guide practice and a particular focus should be directed to antiemetic control in patients receiving such chemotherapy; the assessment of the effectiveness of some 'secondary' antiemetics in this lower emetic risk group may be appropriate. Furthermore, the role of 'secondary' antiemetics (i.e. benzodiazepines, anticholinergics etc.) in combination with 'standard' antiemetics or as rescue medications is not well established, although they are used very frequently in everyday practice, and this may be another area of research focus in the future. An improved generation of such secondary antiemetics may also be a future research endeavour. Cytotoxic drugs delivered by prolonged oral administration such as capecitabine or temozolomide have not had the time course of their associated emesis well documented, and no evidence based recommendations for prophylactic antiemetics can be recommended. A similar situation exists for targeted therapies such as imatininb, which are also administered by prolonged oral dosing. Recording the emesis will require 
modification of the patient diary tools which were initially designed for intermittent dosing. Assessment of nausea/ vomiting should be improved in future studies, and the use of well validated and sensitive multidimensional tools should be carefully considered. Use of the same tool across studies will facilitate in the future the possibility of comparisons. MASCC has developed and validated such a tool [27] and a comprehensive review of the quality and psychometric properties of all tools available to date to measure nausea/ vomiting is available [4].

While the intravenous and oral administration of antiemetics is well established, other delivery systems, such as the granisetron trasdermal patches [3] which are available in some countries such as the USA and Australia, may offer more patient choice, can be convenient, reduce pill counts and be particularly useful where compliance to medication is low, where there are swallowing difficulties or where oral absorption may be compromised. Such delivery systems need further research.

\section{Niche groups}

There are specific treatment groups where evidence is still required about the optimal antiemetic therapy. Patients receiving high dose chemotherapy prior to haemopoietic rescue or multiple day chemotherapy of high emetic potential require better control of emesis. Children's responses to antiemetic combinations should not just be extrapolated from adult studies, because they have a different propensity to vomit, are receiving different regimens, and may respond differently to various classes of antiemetics. The elderly people with cancer should be a special focus of future antiemetic research, considering their comorbidities, polypharmacy, self care abilities, costs, decreased renal and hepatic function affecting drug metabolism and excretion [20].Finally there is a great need for further research into the emesis associated with patients receiving radiation therapy with multiple doses and multiple fields, with and without concomitant chemotherapy.

\section{Non-pharmacological adjuvant interventions}

While the views of clinicians on the use of nonpharmacological approaches to the management of nausea/ vomiting may be mixed, such use as adjuvants to antiemetics may lead to an improved control of symptoms. There is strong evidence that electroacupuncture can significantly improve chemotherapy related nausea/vomiting [12] and that use of acupressure wristbands can have a protective effect in chemotherapy-induced $[12,28]$ and radiotherapy-induced nausea/vomiting [39], although the acupressure results are mixed and their role as single antiemetic interventions in low/minimally emetogenic chemotherapy is unclear. Other potential useful non-pharmacological interventions include hypnosis, dietary management or distraction (particularly in children) that have shown promise, but more concrete evidence from randomised trials is necessary. Cognitive behavioural interventions are appropriate for the management of anticipatory nausea/vomiting, and indeed recommended by MASCC guidelines, although studies in other areas of antiemetic control are almost nonexistent and our understanding of the effects of such treatments needs improvement.

\section{Guidelines}

Studies in new methodologies for guidelines including literature searching, consulting, disseminating and updating are needed. Antiemetic guidelines are published by several groups including MASCC, ASCO and NCCN [22]. The differences in the guidelines often relate to when they are updated and therefore encompass the latest literature. There is very little evidence that has been collected on the efficacy of the dissemination of antiemetic guidelines or their uptake, and their subsequent impact on clinical practice although a prospective study has provided some initial evidence that patients receiving antiemetics based on such guidelines had an improved control of nausea/vomiting compared to those who did not receive guideline-consistent antiemetics, and also there was significant cost savings in the guideline consistent group [29]. A few trials indicate that only around $50 \%$ of patients receive antiemetic prophylaxis as recommended by guidelines. Lack of implementation may be one of the greatest obstacles to effective prophylaxis of nausea and vomiting, because patients do better, when they are receiving guideline recommended antiemetic therapy [11]. More research, however, is required in this area.

MASCC has taken a multinational approach and has standing committees responsible for sections of the guidelines, which can be updated on the web-based version every 6 months, whilst a major revision occurs in an intensive review process every 4 years.

A fruitful research area which is being explored by Cancer Council Australia for guidelines in general, is the use of a Wiki platform. This would allow for wide consultation and dissemination, and guidelines could be updated as new evidence becomes available. It is possible to search the literature electronically for antiemetic trials which could be fed as tare into the wiki as they are published for an expert panel's comments and decision about whether the new evidence warrants a change in the guidelines. Research will be required to see if this is a cost effective method for keeping guidelines constantly updated. 


\section{Conclusions}

Despite the success of the two new classes of antiemetic drugs, the $5 \mathrm{HT}_{3}$ receptor antagonists and the $\mathrm{NK}_{1}$ receptor antagonists in preventing acute and delayed post chemotherapy vomiting there is still much to be achieved in the prediction and treatment of emesis. The understanding of the nature of nausea and more trials to discover how best to control it, are still required. All new drugs and targeted therapies should have their emetic potential defined and the control of emesis with high dose and prolonged oral scheduling requires new studies. More antiemetic trials are needed for radiation induced emesis and in the paediatric age group. Furthermore, combined chemoradiation has become standard therapy of many cancer diseases and randomised antiemetic trials are highly warranted in this setting. The potential of new antiemetics targeted at different receptors such as opioid or mu receptors may see emesis continue to be perceived as less of a problem for patients facing chemotherapy [9].

Conflict of Interest The authors of this paper are all members of Multinational Association of Supportive Care in Cancer (MASCC) but have no financial interest or conflict of interest with regards to the content of this manuscript.

\section{References}

1. Aapro M, Fabi A, Nolè F, Medici M, Steger G, Bachman C, Roncoroni S, Roila F (2010) Double-blind, randomised, controlled study of the efficacy and tolerability of palonosetron plus dexamethasone for 1 day with or without dexamethasone on days 2 and 3 in the prevention of nausea and vomiting induced by moderately emetogenic chemotherapy. Ann Oncol 21:1083-1088

2. Bloechl-Daum B, Deuson RR, Mavros P, Hansen M, Herrstedt J (2006) Delayed Nausea and Vomiting Continue to Reduce Patients'Quality of Life After Highly and Moderately Emetogenic Chemotherapy Despite Antiemetic Treatment. J Clin Oncol 24:4472-4478

3. Boccia RV, Gordan LN, Clark G, Howell JD, Grunberg SM; on behalf of the Sancuso Study Group (2010) Efficacy and tolerability of transdermal granisetron for the control of chemotherapy-induced nausea and vomiting associated with moderately and highly emetogenic multi-day chemotherapy: a randomized, double-blind, phase III study. Support Care Cancer. doi:10.1007/s00520-010-0990-y

4. Brearley SG, Clements CV, Molassiotis A (2008) A review of patient self-report tools for chemotherapy-induced nausea and vomiting. Support Care Cancer 16:1213-1229

5. Burke TA, Wisniewski T, Ernst FR (2010) Resource utilization and costs associated with chemotherapy-induced nausea and vomiting (CINV) following highly or moderately emetogenic chemotherapy administered in the US outpatient hospital setting. Support Care Cancer. doi:10.1007/s00520-009-0797-x

6. Celio L, Frustaci S, Denaro A, et al; for the Italian Trials in Medical Oncology Group (2010) Palonosetron in combination with 1-day versus 3-day dexamethasone for prevention of nausea and vomiting following moderately emetogenic chemotherapy: a randomized, multicenter, phase III trial. Support Care Cancer (in press)

7. Cella D, Eton DT, Fairclough DL, Bonomi P, Hayes AE, Silberman C, Wolf MK, Johnson DH (2002) What is clinically meaningful change on the Functional Assessment Cancer Threrapy - Lung (FACT-L) quesionnaire? Results form Eastern Cooperative Oncology Group (ECOG) Study 5592. J Clin Epidemiol 55:285-295

8. Darmani NA, Janoyan JJ, Crim J, Ramirez J (2007) Receptor mechanism and antiemetic activity of structurally-diverse cannabinoids against radiation-induced emesis in the least shrew. Eur J Pharmacol 563:187-196

9. de Boer-Dennert M, de Wit R, Schmitz PI, Djontono J, v Beurden V, Stoter G, Verweij (1997) Patient perceptions of the side effects of chemotherapy: the influence of the 5-HT3 antagonists. Br J Cancer 76:1055-1061

10. De Mulder PH, Seynaeve C, Vermorken JB, van Liessum PA, Mols-Jevdevic S, Allman EL, Beranek P, Verweij J (1990) Ondansetron compared with high-dose metoclopramide in prophylaxis of acute and delayed cisplatin-induced nausea and vomiting. A multicentre, randomized, double-blind, crossover study. Ann Intern Med 113:834-840

11. Dranitsaris G, Leung P, Warr D (2001) Implementing evidencebased antiemetic guidelines in the oncology setting: results of a 4-month prospective intervention study. Support Care Cancer 9:611-618

12. Ezzo JM, Richardson MA, Vickers A, Allen C, Dibble SL, Issell BF, Lao L, Pearl M, Ramirez G, Roscoe J, Shen J, Shivnan JC, Streitberger K, Treish I, Zhang G (2006) Acupuncture-point stimulation for chemotherapy-induced nausea or vomiting. Cochrane Database Syst Rev 19(2):2285

13. Gore L, Chawla S, Petrilli A, Hemenway M, Schissel D, Chua V, Carides AD, Taylor A, Devandry S, Valentine J, Evans JK, Oxenius B (2009) Aprepitant in adolescent patients for prevention of chemotherapy-induced nausea and vomiting: a randomized, double-blind, placebo-controlled study of efficacy and tolerability. Pediatr Blood Cancer 52:242-247

14. Hainsworth JD (1992) The use of ondansetron in patients receiving multiple-day cisplatin regimens. Semin Oncol 19:48-52

15. Hargreaves R (2002) Imaging substance P receptors (NK1) in the living human brain using positron emission tomography. J Clin Psychiatry 63:18-24

16. Herrington JD, Jaskiewicz AD, Song J (2008) Randomized, placebo-controlled, pilot study evaluating aprepitant single dose plus palonosetron and dexamethasone for the prevention of acute and delayed chemotherapy-induced nausea and vomiting. Cancer 112:2080-2087

17. Hesketh PJ, Aapro M, Street JC, Carides AD (2010) Evaluation of risk factors predictive of nausea and vomiting with current standard-of-care antiemetic treatment: analysis of two phase III trials of aprepitant in patients receiving cisplatin-based chemotherapy. Support Care Cancer 18:1171-1177

18. Hesketh PJ, Kris MG, Grunberg SM, Beck T, Hainsworth JD, Harker G, Aapro MS, Gandara D, Lindley CM (1997) Proposal for classifying the acute emetogenicity of cancer chemotherapy. J Clin Oncol 15:103-109

19. Hickok JT, Roscoe JA, Morrow GR (2001) The role of patients' expectations in the development of anticipatory nausea related to chemotherapy for cancer. J Pain Symptom Manage 22:843-850

20. Jakobsen JN, Herrstedt J (2009) Prevention of chemotherapyinduced nausea and vomiting in elderly cancer patients. Crit Rev Oncol Hematol 71:214-221

21. Jiang J, Bunda JL, Doss GA, Chicchi GG, Kurtz MM, Tsao KL, Tong X, Zheng S, Upthagrove A, Samuel K, Tschirret-Guth R, Kumar S, Wheeldon A, Carlson EJ, Hargreaves R, Burns D, 
Hamill T, Ryan C, Krause SM, Eng W, DeVita RJ, Mills SG (2009) Potent, brain-penetrant, hydroisoindoline-based human neurokinin-1 receptor antagonists. J Med Chem 52:3039-3046

22. Jordan K, Sippel C, Schmoll H-J (2007) Guidelines for Antiemetic Treatment of The Oncologist 12: 1143-1150

23. Kaiser R, Sezer O, Papies A, Bauer S, Schelenz C, Tremblay PB, Possinger K, Roots I, Brockmöller J (2002) Patient-tailored antiemetic treatment with 5-hydroxytrptamine type 3 receptor antagonists according to cytochrome P-450 2wD6 genotypes. J Clin Oncol 20:2765-2767

24. Keire DA, Bowers CW, Solomon TE, Reeve JR Jr (2002) Structure and receptor binding of PYY analogues. Peptides 23:305-321

25. Lajolo PP, de Camargo B, del Giglio A (2009) Omission of day 2 of antiemetic medications is a cost saving strategy for improving chemotherapy-induced nausea and vomiting control: results of a randomized phase III trial. Am J Clin Oncol 32:23-26

26. Martin CG, Rubenstein EB, Elting LS, Kim YJ, Osoba D (2003) Measuring chemotherapy-induced nausea and emesis. Cancer 98:645-655

27. Molassiotis A, Coventry PA, Stricker CT, Clements C, Eaby B, Velders L, Rittenberg C, Gralla RJ (2007) Validation and psychometric assessment of a short clinical scale to measure chemotherapy-induced nausea and vomiting: the MASCC antiemesis tool. J Pain Symptom Manage 34:148-159

28. Molassiotis A, Helin AM, Dabbour R, Hummerston S (2007) The effects of P6 acupressure in the prophylaxis of chemotherapyrelated nausea and vomiting in breast cancer patients. Complement Ther Med 15:3-12

29. Molassiotis A, Saunders MP, Valle J, Wilson G, Lorigan P, Wardley A, Levine E, Cowan R, Loncaster J, Rittenberg C (2008) A prospective observational study of chemotherapy-related nausea and vomiting in routine practice in a UK cancer centre. Support Care Cancer 16:201-208

30. Molassiotis A, Stricker CT, Eaby B, Velders L, Coventry PA (2008) Understanding the concept of chemotherapy-related nausea: the patient experience. Eur J Cancer Care 17:444-453

31. Molassiotis A, Yam BM, Yung H, Chan FY, Mok TS (2002) Pretreatment factors predicting the development of postchemotherapy nausea and vomiting in Chinese breast cancer patients. Support Care Cancer 10:139-145

32. Morrow GR (1985) The effect of susceptibility to motion sickness on the side effects of cancer chemotherapy. Cancer 55:2766-2770

33. Navari RM, Einhorn LH, Snr LPJ, Passik SD, Vinson J, McClean J, Chowhan N, Hanna NH, Johnson CS (2007) A phase II trial of olanzapine, dexamethasone, and palonosetron for the prevention of chemotherapy-induced nausea and vomiting. Support Care Cancer 15:1285-1291

34. Okumura M, Arakawa R, Ito H, Seki C, Takahashi H, Takano H, Haneda E, Nakao R, Suzuki H, Suzuki K, Okubo Y (2008) Suhara T (2008) Quantitative analysis of NK1 receptor in the human brain using PET with 18 F-FE-SPA-RQ. J Nucl Med 49:1749-175

35. Olver IN, Taylor AE, Whitford H (2005) Relationships between patients' pre-treatment expectations of toxicities and post chemotherapy experiences. Psycho-oncol 14:25-33

36. Osoba D (2000) Health-related quality-of-life assessment in clinical trials of supportive care in oncology. Support Care Cancer 8:84-88

37. Pirri C, Katris P, Trotter J, Bayliss E, Bennett R, Drummond P (2010) Risk factors at pretreatment predicting treatment-induced nausea and vomiting in Australian cancer patients: a prospective, longitudinal, observational study. Support Care Cancer. doi:10.1007/s00520-010-0982-y

38. Pollera CF, Gimelli D (1989) Prognostic factors influencing cisplatin induced emesis. Definition and validation of a predictive logistic model. Cancer 64:1117-1122

39. Roscoe JA, Bushunow P, Jean-Pierre P, Heckler CE, Purnell JQ, Peppone LJ, Chen Y, Ling MN, Morrow GR (2009) Acupressure bands are effective in reducing radiation therapy-related nausea. $\mathrm{J}$ Pain Symptom Manage 38:381-389

40. Roscoe JA, Morrow GR, Colagiuri B, Heckler CE, Pudlo BD, Colman L, Hoelzer K, Jacobs A (2010) Insight in the prediction of chemotherapy-induced nausea. Support Care Cancer 18:869-876

41. Rudd JA, Cheng CH, Naylor RJ, Ngan MP, Wai MK (1999) Modulation of emesis by fentanyl and opioid receptor antagonists in Suncus murinus (house musk shrew). Eur J Pharmacol 374:7784

42. Soukoup M (1996) Management of cyclophosphamide-induced emesis over repeated courses. Oncology 53:39-45

43. Sullivan JR, Leyden MJ, Bell R (1983) Decreased cisplatininduced nausea and vomiting with chronic alcohol ingestion. New Engl J Med 309:796

44. Tan L, Liu J, Liu X, Chen J, Yan Z, Yang H, Zhang D (2009) Clinical research of Olanzapine for prevention of chemotherapyinduced nausea and vomiting. J Exp Clin Cancer Res 28:131

45. Tramèr MR, Carroll D, Campbell FA, Reynolds DJ, Moore RA, McQuay HJ (2001) Cannabinoids for control of chemotherapy induced nausea and vomiting: quantitative systematic review. BMJ 323:16-21

46. Tremblay PB, Kaiser R, Sezer O, Tremblay PB, Kaiser R, Sezer O, Rosler N, Schelenz C, Possinger K, Roots I, Brockmoller J (2003) Variations in the 5hydroxytryptamine type 3B receptor gene as predictors of the efficacy of antiemetic treatment in cancer. J Clin Oncol 21:2147-2155

47. Van Sickle MD, Oland LD, Mackie K, Davison JS, Sharkey KA (2003) Delta9-tetrahydrocannabinol selectively acts on CB I receptors in specific regions of dorsal vagal complex to inhibit emesis in ferrets. Am J Physiol Gastrointest Liver Physiol 285:566-57

48. Vardy J, Chiew KS, Galica J, Pond GR, Tannock IF (2006) Side effects associated with the use of dexamethasone for prophylaxis of delayed emesis after moderately emetogenic chemotherapy. $\mathrm{Br}$ J Cancer 94(7):1011-1015

49. Warel MA, Daeninck P, Maida V (2008) A review of nabilone in the treatment of chemotherapy-induced nausea and vomiting. Ther Clin Risk Manag 4:99-107

50. Warr DG, Street JC, Carides AD (2010) Evaluation of risk factors predictive of nausea and vomiting with current standard-of-care antiemetic treatment: analysis of phase 3 trial of aprepitant in patients receiving adriamycin-cyclophosphamide-based chemotherapy. Support Care Cancer. doi:10.1007/s00520-010-0899-5

51. Yakabi K, Sadakane C, Noguchi M, Ohno S, Ro S, Chinen K, Aoyama T, Sakurada T, Takabayashi H, Hattori T (2010) Reduced ghrelin secretion in the hypothalamus of rats due to cisplatininduced anorexia. Endocrinology 151:3773-3782

52. Yeh YC, McDonnell A, Klinger EV, Fowler B, Matta L, Voit D, Reddy P (2010) Comparison of healthcare resource use between patients receiving ondansetron or palonosetron as prophylaxis for chemotherapy-induced nausea and vomiting. J Oncol Pharm Pract. doi:10.1177/1078155210366491 